\title{
Oceanic APNT Using Air-to-Air Ranging
}

\author{
Okuary Osechas ${ }^{1}$, Thomas Gräupl ${ }^{1}$, and Gerhard Berz ${ }^{2}$ \\ ${ }^{1}$ German Aerospace Center (DLR), Oberpfaffenhofen, Germany \\ ${ }^{2}$ EUROCONTROL, Brussels, Belgium
}

\begin{abstract}
This paper describes a new solution for alternative navigation services in oceanic airspace, using collaborative methods, enabled by air-to-air ranging in future navigation technologies. Improving the performance of alternative services is particularly desirable in oceanic airspace, as the demand for slots in the existing organized track systems starts approaching their capacity. The results show how this technology can help support a single aviation user suffering an on-board outage of satellite navigation, or how it could bridge a large-scale regional outage up to $3000 \mathrm{~km}$ wide.
\end{abstract}

\section{Introduction}

The North Atlantic Organized Track System (NAT-OTS) is a set of flight routes that connect the European and North American airspaces. More than two thousand aircraft cross the Atlantic Ocean every day [1] and the number is set to continue growing in the foreseeable future [2. The airspace capacity along the NAT-OTS is limited by the required separation minima between aircraft. While Global Navigation Satellite Systems (GNSS) have enabled Performance-Based Navigation (PBN) services that would support much smaller minima over the ocean, current procedures do not reflect this improved performance. A key element is the lack of an alternate means of radionavigation, in the event of a large-scale outage of GNSS.

The development of navigation services based on GNSS has increased the achievable navigation performance along these trans-oceanic routes and, in principle, this setup would support an increase in traffic density; however, the lack of an alternate position, navigation and timing (APNT) system leaves aircraft to failures in GNSS-based navigation services. The reliance on inertial navigation as backup to GNSS has led to greater separation between aircraft over the ocean $(50 \mathrm{NM})$ than over land $(5 \mathrm{NM})$. Aircraft separation cannot be substantially reduced without an APNT capability.

In this paper we show how Air-to-Air (A2A) ranging, potentially a component of the future L-Band Digital Aeronautical Communication System (LDACS), could provide an alternate navigation capability and, furthermore, we show how this capability would reflect in the ability to bridge large-scale outages in GNSS-based services. The implications of A2A-enabled positioning for APNT over land were first discussed in [3], where en-route aircraft support other users, for example on approach, by improving their positioning in the terminal area with A2A ranging measurements. However, the method that enables APNT in the terminal area, based on A2A ranging, is markedly different from the one that provides oceanic coverage.

\section{Motivation}

An alternative to GNSS-based navigation, capable of providing substantial improvement over the performance of inertial systems, would enable a substantial reduction in separation minima along the NAT-OTS. The current situation is that with no Air Traffic Control (ATC) services available, NAT-OTS users rely solely on procedural control.

The reduction of separation minima would, in turn, significantly increase the traffic-handling capacity of the NATOTS. According to the appropriate manual 4 " It is important to consider that the limited economical height (Flight 
Levels 310-400), in combination with the large separation requirements, leads to congestion at peak hours".

In the scope of a changing CNS environment, this paper presents a look at the navigation capabilities enabled by A2A ranging. However, the gains in navigation performance are only meaningful in combination with other improvements in the oceanic CNS environment, such as Space-Based ADS-B and oceanic communication services, whether satellite-based or A2A.

The method described in this paper would provide navigation services for the NAT-OTS, even during a large-scale outage of GNSS-based services. This method is based on the assumption an A2A ranging capability exists, both for data transmission and for ranging. It seems plausible that the LDACS will include this capability, but the methods in this paper are still applicable if the physical layer implementation is not based on LDACS.

Furthermore, given that a process has been initiated, to standardize LDACS as a communications system for aviation, we also use the opportunity to suggest tweaks and extensions to the protocol that can be considered in this process. One such element could be an "oceanic" flavour to LDACS that would better support A2A navigation over long distances.

\section{Background}

This section introduces the concepts required to discuss the performance of A2A positioning for APNT. For many authors APNT services are assumed to be provided by terrestrial infrastructure, with Distance Measuring Equipment (DME) sometimes assumed to be the likely source of APNT in the short term. In contrast, we propose a method that requires no terrestrial infrastructure.

\section{Performance-Based Navigation and Separation Minima}

The main idea behind PBN is that navigation services can be specified in terms of measures like accuracy and integrity, rather than in terms of the configuration of available sensors. The first development in PBN was area navigation (RNAV), which defines specific requirements for the navigation system that are supported by a variety of technology, both satellite-based and terrestrial. Required Navigation Performance (RNP), on the other hand, is a class of services enabled by GNSS; RNP allows pilots greater flexibility in choosing their routing, with the hope of making more efficient use of the airspace.

The key concept behind RNP is that the airborne navigation equipment needs to ensure that the position estimates it computes are compliant with the RNAV/RNP Type required by a particular operation. The RNAV/RNP Type is usually by a number that indicates the 95th percentile of the horizontal component of the Total System Error (TSE), expressed in nautical miles (NM).

The TSE is made up of several contributions: the Path Definition Error (PDE) corresponds to imperfections in how a flight path is specified; the Flight Technical Error (FTE) corresponds to the ability of the flight controller to follow a prescribed trajectory:

$$
T S E^{2}=P D E^{2}+F T E^{2}+N S E^{2} .
$$

The Navigation System Error (NSE) accounts for the uncertainty in the position estimate. For RNAV and RNP services of the same type indicator, the NSE requirement will be the same. For most RNP operations the PDE is assumed to be negligibly small and the FTE is bounded from standard reference values. That leaves the NSE as the performance criterion that the navigation system needs to fulfill, so as to accommodate the budgeted FTE and keep the TSE below the RNP Type indicator.

For oceanic routes aircraft are required to have "at least two fully serviceable Long Range Navigation Systems" (LRNS) 4. Typically this amounts to having GNSS and inertial navigation available, where the inertial system will support RNAV 10 performance over the typical distances of a transatlantic crossing. 


\begin{tabular}{||c|ccc||}
\hline RNP Type & TSE & NSE & Minimum Separation \\
\hline 10 & $18.52 \mathrm{~km}(10 \mathrm{NM})$ & $18.50 \mathrm{~km}$ & $92.600 \mathrm{~km}(50 \mathrm{NM})$ \\
4 & $7.41 \mathrm{~km}(4 \mathrm{NM})$ & $7.35 \mathrm{~km}$ & $55.56 \mathrm{~km}(30 \mathrm{NM})$ \\
1 & $1.85 \mathrm{~km}(1 \mathrm{NM})$ & $1.60 \mathrm{~km}$ & $9.260 \mathrm{~km}(5 \mathrm{NM})$ \\
0.3 & $0.556 \mathrm{~km}(0.3 \mathrm{NM})$ & $0.31 \mathrm{~km}$ & $5.556 \mathrm{~km}(3 \mathrm{NM})$ \\
\hline
\end{tabular}

Table 1: Maximum NSE and minimum separation distance supported by different RNP types.

\section{Air-to-Air Radio Links: LDACS}

The current vision in developing LDACS as a new safety-of-life communications service in Air Traffic Management (ATM) includes the ability of LDACS to provide ranging measurements [5], and its ability to provide direct interaircraft radio links 6. In the past, the vision of an A2A ranging capability has been shown to bring significant benefit to Alternative Position, Navigation and Timing (APNT) services 3 .

The key assumptions about LDACS, in the argument of this paper, are: its ability to provide range measurements between aircraft and its ability to provide minimal protocol-level information that will facilitate navigation services. Given that, for pseudoranging configuration, LDACS has been shown to support a measurement error with standard deviation of $\sigma_{\rho}=6 \mathrm{~m}$, we assume that the equivalent error for two-way ranging is $\sigma_{r}=8 \mathrm{~m}$, which approximates an increase by a factor of $\sqrt{2}$.

\section{Fault Models}

For the purposes of quantifying the benefits of the APNT service we analyze the effects of two types of faults that can affect GNSS services: one is a small-scale, single user fault, the other is a large-scale regional fault. In the single-user case we model an individual aircraft as losing GNSS services, without making any assumptions on the origin of the fault. In the case of the wide-area service outage we model a "blackout" region over the North Atlantic as being GNSS denied. Neither fault model makes any assumptions on the possible underlying fault mechanism; a single-user fault could, for example, be due to a hardware failure, while the large-scale outage could be originated by atmospheric effects or from radio interference (inentional or unintentional) or from some other source.

\section{Single-Aircraft Fault}

In a densely-packed cluster of aircraft along the NAT-OTS, loss of GNSS service even for a single user could become a disruption, if the performance of the alternative PNT system degrades over time. The ability to support such a user with A2A ranging would, therefore, bring substantial benefit to the logistics of oceanic ATM.

In the experiments for this paper we analyze a single flight, traversing the NAT-OTS eastbound. Along the entire track we record the NSE and monitor its compliance with RNP 4.

\section{Large-Scale GNSS Outage}

A different scenario in which A2A promises benefits is a large-scale outage of GNSS services. While we make no assumption regarding origin of such a fault, several fault mechanisms are conceivable that could lead to such disruptions, including ionospheric scintillations and radio interference events [7].

Given that the track system is longer than wide, we can model the tracks as a straight line, or a geodesic great circle, and the results are comparable. The key parameter is the distance between the point at which the tracks enter the blackout zone and where they exit. 


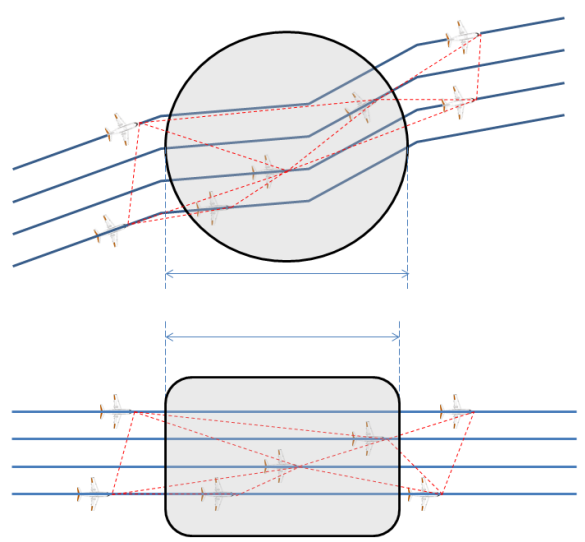

(a)

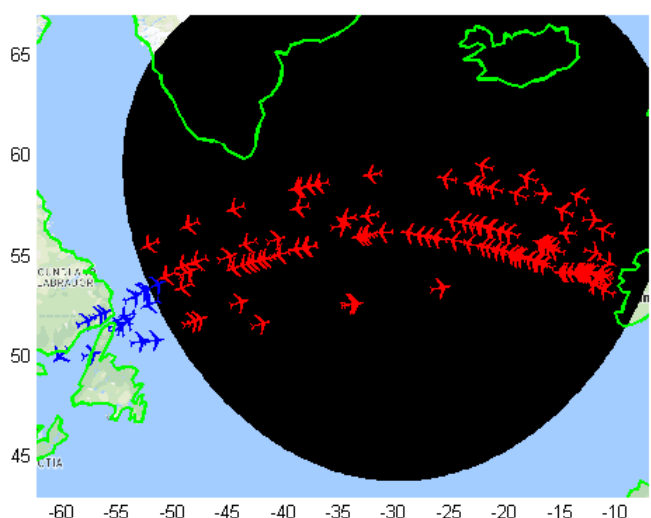

(b)

Figure 1: (a) The NAT-OTS is organized by segments of great circles, but for the purposes of the paper the difference between segments of great circles, great circles and straight lines is assumed to be negligibly small. (b) A blackout zone with a radius of $1500 \mathrm{~km}$ represents $\approx 3000 \mathrm{~km}$ of outage along the NAT-OTS. Aircraft in blue are assumed to have active GNSS position solutions, aircraft indicated in red are modeled as denied.

\section{State of the Art and Current Developments}

As specified in the standard [4, an aircraft must be equipped with a GNSS receiver and an inertial system to use the NAT-OTS. The service type provided is typically considered to be RNAV/RNP 10, as a GNSS outage would lead to an increase in the horizontal error due to inertial coast; according to the standard this error must be expected to grow at a rate of $8 \mathrm{NM} / \mathrm{h}[\underline{8}$.

For the purposes of safe operations an aircraft entering the NAT-OTS must, therefore, assume that an outage of GNSS will occur en-route, and maintain adequate separation from other traffic. As discussed above, the reasons for this assumption stems from the fact that oceanic operations are conducted without surveillance services.

In the broader context of the ATM paradigm shift towards PBN, the ability of supporting tighter error bounds in oceanic operations would, eventually, also lead to increased capacity. Providing an alternate capability to GNSSbased positioning could, therefore, have a beneficial impact on the efficient use of transatlantic or, more generally, oceanic airspace.

\section{Navigation Algorithm}

A user located at a position $\mathbf{x}$ aims to compute a position solution $\hat{\mathbf{x}}$, preferably from GNSS-based measurements $\mathbf{y}_{G}$, which will have a position error distributed with $\varepsilon_{G} \sim \mathcal{N}\left(0, \boldsymbol{\Sigma}_{G}\right)$. Should GNSS become unavailable the user reverts to A2A-based measurements $\mathbf{y}_{A}$ to compute position estimate with a different distribution: $\varepsilon_{A} \mathcal{N}\left(0, \boldsymbol{\Sigma}_{A}\right)$.

For the purposes of this paper the equations assume that two-way ranging is available. In the particular case of LDACS-based ranging, this capability is envisioned for future versions, but is not yet part of the standard. The range between an aircraft aiming to compute its own position estimate and airborne ranging source $k$ is:

$$
r^{(k)}=\left(\mathbf{x}_{u}-\mathbf{x}^{(k)}\right) \cdot \mathbf{1}^{(k)}+T^{(k)}+M^{(k)}+\varepsilon^{(k)}
$$

Unlike in satellite or terrestrial positioning, in A2A setups the position uncertainty of an aerial ranging source is typically in a similar order of magnitude as the ranging error. The position uncertainty can be thought of as an error in the ephemeris of the ranging source, which is described by adding the noise on the range measurement $\sigma_{r}$ to the position uncertainty on the ranging source along the LoS. The ranging error at user $k$, resulting from ranging noise 
$\sigma_{r}$ and position error at receiver $j$ can be modeled as:

$$
\sigma_{k, j}^{2}=\sigma_{r}^{2}+\left\|\boldsymbol{\Sigma}_{j} \cdot \mathbf{1}_{k, j}\right\| .
$$

In the context of RNP operations, the effects of tropospheric delays and multipath can be considered as negligibly small, as they typically induce random errors that orders of magnitude smaller than $\sigma_{r}$.

Position estimates from ranging measurements can be computed using a Gauss-Newton-based method similar to that employed in satellite navigation [9]. In such a case, the user performs an iterative search for the position that minimizes the estimation residual.

The residual minimization algorithm can be implemented iteratively, where an initial position guess $\mathbf{x}_{0}$ is computed, which in turn leads to expected measurements $\mathbf{r}_{0}$. In his iterative setup a geometry matrix $\mathbf{G}_{i}$ needs to be recomputed for each successive iteration, such that a position correction $\delta \mathbf{x}$ can be obtained from comparing expected measurements with actual measurements:

$$
\delta \mathbf{r}_{i}=\mathbf{r}-\mathbf{r}_{i} .
$$

This mismatch between expected and actual measurements can be used to improve the position estimate in the next iteration step, by applying the weighted pseudoinverse of the geometry matrix to the residuals vector:

$$
\delta \mathbf{x}_{i+1}=\left(\mathbf{G}^{T} \mathbf{W G}\right)^{-1} \mathbf{G}^{T} \mathbf{W} \delta \mathbf{r}_{i} .
$$

Once the algorithm converges to $\left\|\mathbf{x}_{i+1}-\mathbf{x}_{i}\right\| \leq \varepsilon$, where $\varepsilon>0$, the convergence value becomes the position estimate

$\hat{\mathbf{x}}$, which has a distribution that can be overbounded with a multi-variate Gaussian $\mathcal{N}(0, \boldsymbol{\Sigma})$, where $\boldsymbol{\Sigma}=\left(\mathbf{G}^{T} \mathbf{W G}\right)^{-1}$.

The known positions of the ranging sources are combined with an assumed position of the user receiver that leads to a set of line-of-sight $(\mathrm{LoS})$ unit vectors $\mathbf{1}^{k}$. These vectors make up the geometry matrix $\mathbf{G}$, which iteratively guides the search for the best position estimate [9].

\section{Positioning Performance}

A useful performance metric in assessing the benefits of A2A ranging is the horizontal component of the positiondomain error, equivalent to the NSE in equation (7). As a model for the covariance matrix $\boldsymbol{\Sigma}$, we can use:

$$
\boldsymbol{\Sigma}=\left(\mathbf{G}^{T} \mathbf{W G}\right)^{-1}
$$

The model in equation (6) allows assessing the NSE as a function of the positions of peer aircraft, simply by taking the trace of the East-North submatrix of $\boldsymbol{\Sigma}$ :

$$
N S E=\sqrt{\boldsymbol{\Sigma}_{1,1}+\boldsymbol{\Sigma}_{2,2}} .
$$

The collaborative nature of A2A positioning requires that a set of airborne users have positioning services available. For the computations in this paper, in accordance with the fault model described above, we assume that a set of users exists, which have GNSS-based positioning available. The positioning uncertainty for these "active" users is smaller than the achievable accuracy for APNT-only users. These active users have the most accurate position solutions available and, by becoming A2A ranging sources enable other users to compute position solutions.

For this reason we discuss the results of the method in terms of three different types of users: active, enabled and denied. Active users have an active GNSS positioning system; enabled users have positioning services available, enabled through A2A ranging; while denied users continue to have no position solution available.

The aim of our method is to minimize the number of denied users in the presence of a large-scale outage of GNSS services. 


\section{Performance Degradation due to Multihop Positioning}

The scenario where each airborne ranging source computes its position estimate from external sources (GNSS, terrestrial sources) is often referred to as a single-hop situation. As an example the terminal-area setup in [3] considers only single-hop A2A positioning. On the contrary, airborne users of A2A positioning can, in turn, become A2A ranging sources, provided their position estimates are sufficiently accurate. Such a case can be referred to as multi-hop, as airborne sources exist that do not have a direct link to external systems.

There is a degradation in performance that increases with every hop, as modeled in equation (3). The challenge in meaningfully covering a large-scale GNSS outage lies in having a network of A2A participants that is sufficiently dense to support the desired NSE at the extremes.

\section{Performance Evaluation}

The assessment of whether a proposed A2A ranging protocol provides sufficient performance for oceanic APNT requires checking the expected NSE against the desired containment area associated with the navigation service. As a baseline case we assume that it is desirable to provide RNP 4 over the NAT-OTS, during a large-scale GNSS outage.

For a given configuration it is possible to compute the maximum fault radius that would still be covered by the APNT system. The configuration includes a geographic distribution of aircraft and assumptions about the underlying A2A ranging protocol, most prominently coverage distance $D$ and ranging accuracy $\sigma_{r}$.

In this section we compute the maximum fault radius that can be bridged with realistic traffic patterns, assuming that the LDACS A2A ranging protocol will resemble the more conventional air-to-ground version of LDACS.

\section{Data Source}

The performance results of our method depend heavily on the specific distribution of aircraft traffic, for that reason we aim to use traffic data that is as realistic as possible. Given the difficulties associated with obtaining real surveillance data for the NAT-OTS we resort to simulated data, for which we use the air traffic simulator FACTS2. This simulator is based on historic flight plan data and has been validated against real-world surveillance data [1] to support experiments where the fidelity of the aircraft position can tolerate mismatches in the order of tens of kilometers.

\section{Results for Realistic Traffic}

As a way to show the real-world relevance of the positioning algorithm enabled by A2A ranging we test the method on the data set described above. First we test two single-aircraft outages, then we test a large-scale regional outage. For these tests we assume a baseline configuration of LDACS, where specific modifications are introduced to tackle certain aspects of the problem.

The baseline LDACS model assumes a coverage radius of $370 \mathrm{~km}(\approx 200 \mathrm{NM})$, with a ranging noise of $8 \mathrm{~m}$. The coverage radius is the same as currently envisioned for G2A LDACS, while the ranging error stems from assuming that the two-way ranging error is equivalent to $\sqrt{2}$ times the one-way ranging error, typically modeled as being $6 \mathrm{~m}[5]$.

Users with GNSS-active users are assumed to have a position covariance matrix $\boldsymbol{\Sigma}=\left[\begin{array}{ccc}2^{2} & 0 & 0 \\ 0 & 2^{2} & 0 \\ 0 & 0 & 3^{2}\end{array}\right] m^{2}$, which corresponds to an optimistic multi-frequency multi-constellation position fix. 


\begin{tabular}{l|l} 
Parameter & Value \\
\hline LDACS Coverage Distance $(D)$ & $370.4 \mathrm{~km}(200 \mathrm{NM})$ \\
LDACS Ranging Error $\left(\sigma_{r}\right)$ & $8 \mathrm{~m}$ \\
Target Service & RNP 4
\end{tabular}

Table 2: Configuration of simulation parameters for performance baseline.

\section{Single-User Outage}

In a situation where a single airborne user loses GNSS service, the notion that other aircraft will support the affected peer is viable, as long as a sufficient number of neighboring GNSS-enabled peers are available within the distance of one LDACS coverage radius. To assess the feasibility of providing RNP 4 during such a crossing of the NAT-OTS we use the aforementioned simulated traffic data.

From the data we pick a single user, traversing the NAT-OTS eastbound. The aircraft leaves Newfoundland at approximately 00:00 UTC and arrives in Ireland at 03:30 UTC, surrounded by a number of other aircraft. We consider this to be situation that is representative of a typical day, where a large proportion of eastbound traffic occurs during the night hours.

In the simulated traffic data we select two aircraft for closer inspection. The main difference between them is that one is surrounded by GNSS-enabled users, while the other is on the outside track. The aircraft on the outside track has measurements available from its left side, which is asymmetric. Intuitively this may seem like it would lead to a significant degradation in positioning performance, but the results in Fig. 2 show that the degradation with respect to the other user is negligibly small, compared to the magnitude of the error.

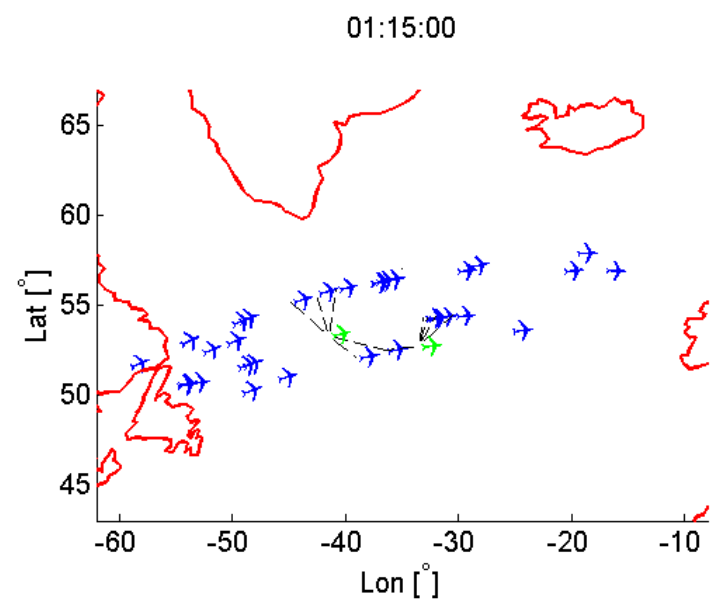

(a)

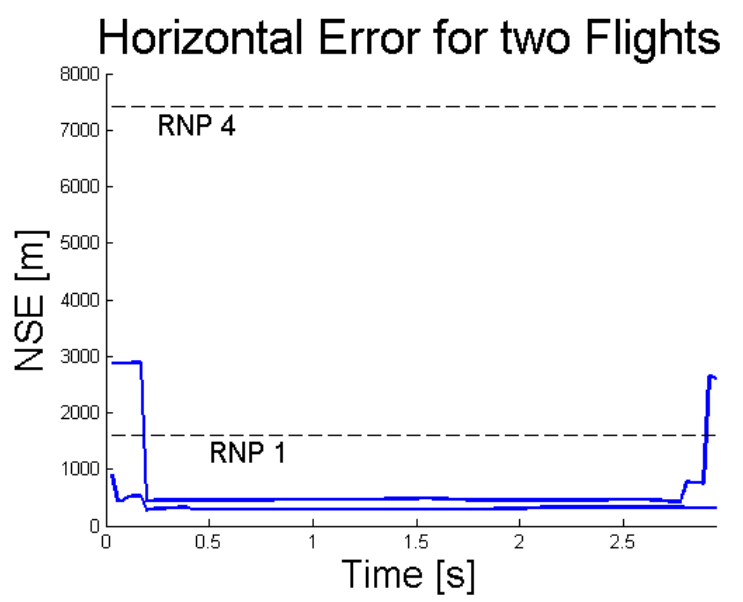

(b)

Figure 2: (a) The set of aircraft crossing the NAT-OTS at 01:15 UTC, where the two A2A-enabled users are highlighted in green and their A2A links indicated with dashed lines (b) The error performance for the two affected aircraft is compliant with RNP 4.

The plot of Fig. 2 (b) shows the position error for the two highlighted aircraft. One key message is that in both cases the NSE stays below the requirement for RNP 4 at all times. In fact, except for the initial and final transient periods, the errors are compliant even with RNP 1. These transients can be explained as simulation artifacts, as no aircraft are defined behind the two affected aircraft at 00:00 UTC, when in a real-world environment other traffic would be following. After 15 mins, this traffic does come into the scope of the simulation and both NSEs fall below $1600 \mathrm{~m}$.

This behavior highlights the utility of A2A ranging for aiding oceanic users in case of an on-board GNSS outage. In this situation the ATM system would function seamlessly, even under an RNP 4 regime. State-of-the-art technology 


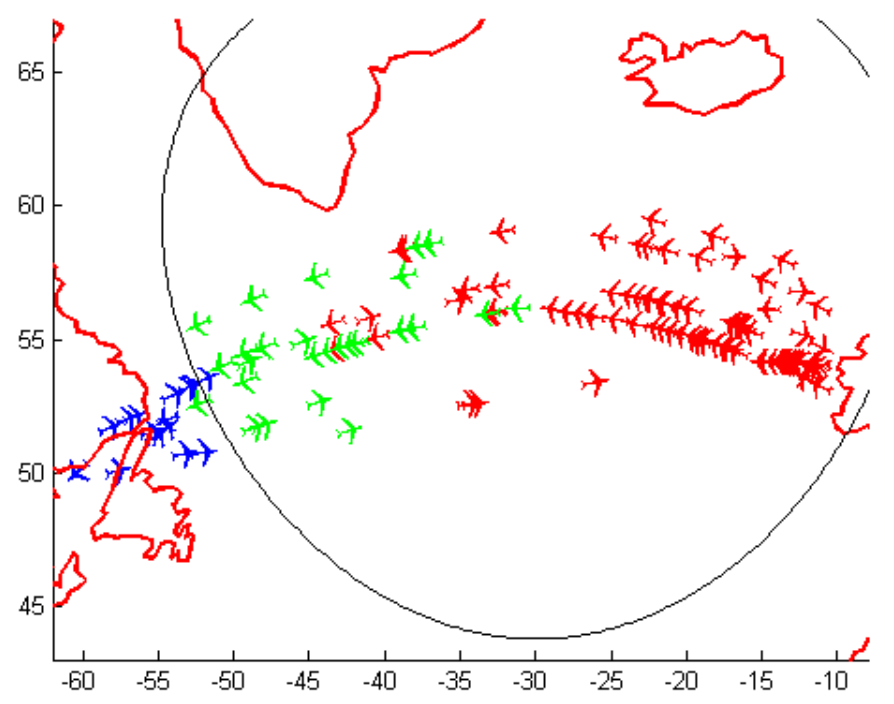

Figure 3: Trying to bridge $3000 \mathrm{~km}$ worth of outage along the NAT-OTS with LDACS. Blue aircraft have an active GNSS-based position fix, red aircraft have no service and green aircraft have a position fix enabled by A2A positioning.

would not support this kind of performance across the entire oceanic crossing.

Finally, it should be noted that the LDACS coverage radius for this scenario was left at the baseline value of $370 \mathrm{~km}$ (200 NM), as indicated in Table 2

\section{Large-Scale Outage}

Applying the previously discussed collaborative navigation method to the large-scale outage we obtain results that depend heavily on the assumed coverage radius for LDACS A2A.

For the baseline case we obtain that faults of up to $1500 \mathrm{~km}$ can be bridged under typical traffic loads, provided that GNSS-enabled aircraft are available on both sides of the fault. In cases where the bridge is only "fastened" on one side, like in Fig. 3, it is more difficult to provide coverage.

\section{Improving Coverage}

A first observation from Fig. 3 is that the standard coverage area envisioned for terrestrial LDACS will not be sufficient to support oceanic RNP 4. Two modifications to the status quo could enable all aircraft with A2A-based position solutions: increasing the coverage of the LDACS ranging signal and modifying the trajectory of the aircraft, so as to ensure adequate positioning throughout the entire transatlantic journey.

\section{Increasing LDACS Coverage Distance}

Increasing the coverage distance of the ranging technology, specifically for oceanic operations, provides the method with increased flexibility. A cluster of aircraft can provide collaborative position estimates with greater flexibility, with respect to the relative location of the participants. The collaborative method would support greater accuracy, but also a wider range of aircraft densities. 
It seems evident that increasing the coverage distance of LDACS would also improve the availability of A2A-based RNP services. The relationship between coverage and availability, however, depends on the geometry of the aircraft over the NAT-OTS.

In terms of service provision, increasing the coverage radius of LDACS leads to more aircraft having RNP 4 available than in the baseline case of Fig. 3. In the plots of Fig. 4 we show the effects of increasing $D$ by a factor of two and four, respectively.

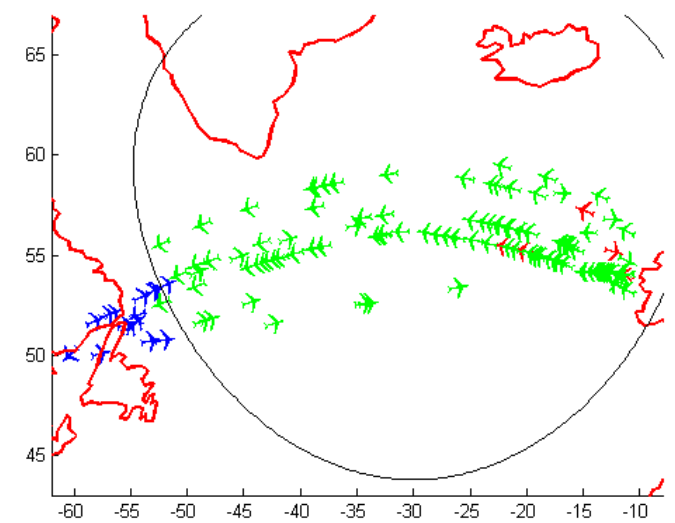

(a)

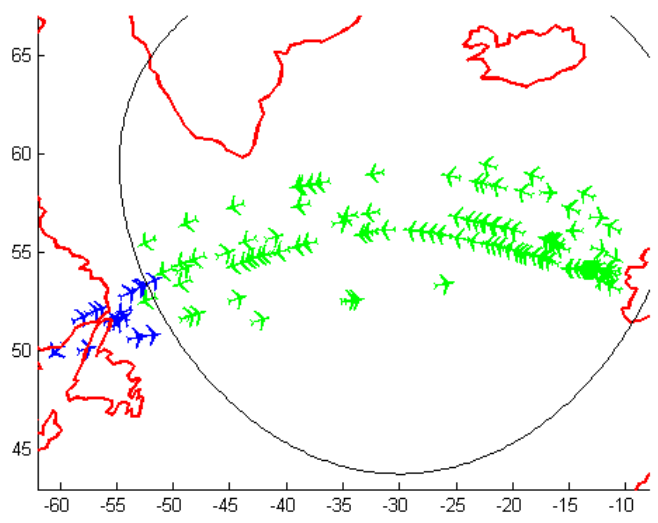

(b)

Figure 4: (a) Increasing $D$ by a factor of two services nearly all traffic. (b) The increase by a factor of four does provide RNP 4 to all simulated aircraft.

In terms of interference with other systems, concern over the increased transmit power, required for increased coverage, will have to be addressed. An "oceanic flavor" of LDACS will only be feasible, if the resulting out-of-channel interference with existing systems. Given that the oceanic environment is characterized by a lack of infrastructure, it seems more feasible to increase transmit power over water than over land. The most challenging situation would likely arise with terrestrial infrastructure near the coastline, where the complexity of adequate frequency planning would increase.

\section{Active Shaping of Aircraft Traffic}

The details in Fig. 3 suggest that aircraft flying in isolation are less likely to have A2A positioning available, than aircraft flying in proximity of other aircraft. The immediate syllogism is that aircraft flying in dense formation will provide the most reliable navigation capability.

Designing transatlantic trajectories in such a way that A2A positioning performance is maximized is a more complex approach, but we show below that it would significantly increase the capacity of the NAT-OTS. Some of the ATM technology required for such a complex setup is still under development, for example required time of arrival (RTA) or similar services would be useful [10], but basic planning can already be provided by the oceanic clearance process, which assigns aircraft a particular track, a specific time for accessing the track and a required velocity [4. In the very least, adequate clearance design and coordination, in compliance with current standards, would avoid individual aircraft being left out from A2A APNT support, but future oceanic operations will likely continue to adapt demand and capacity balancing [1].

To assess the potential capacity increase we modify the fault model, to better illustrate the reasoning behind this method. We assume that aircraft fly across the NAT-OTS in strict formation, with the lateral separation between 
tracks given by 4 as $0.5^{\circ}$ latitude and the longitudinal separation set to $30 \mathrm{NM}$, as in Table 1 .

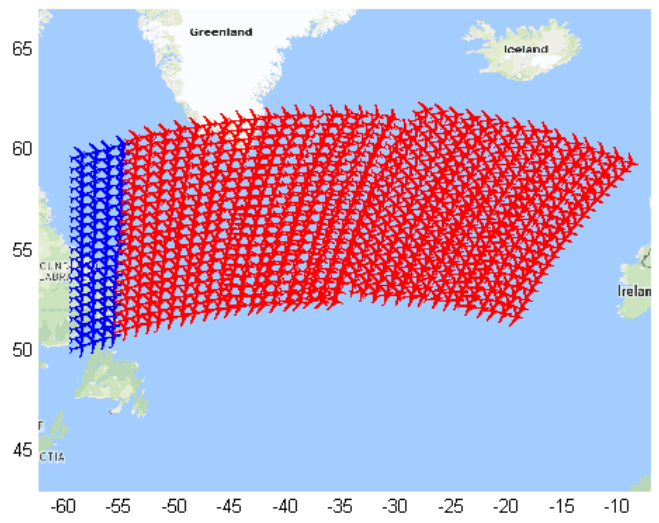

(a)

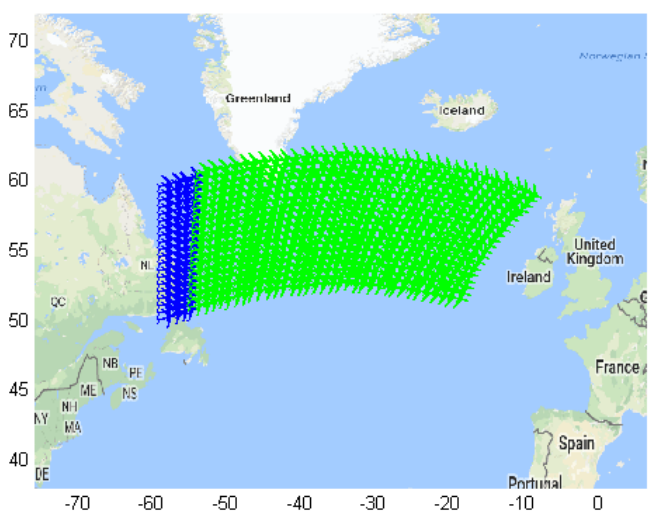

(b)

Figure 5: (a) Synthetic scenario: Ten tracks of aircraft fly with $30 \mathrm{NM}$ along-track separation. The four western-most rows of aircraft are GNSS enabled, the rest are denied. (b) The proposed A2A capability would provide RNP 4 to 680 aircraft in the NAT-OTS simultaneously.

The synthetic case can further be simplified by approximating great circles as straight lines. As assumed before, the impact of this simplification on the position error is negligibly small, compared to the error influences of the other effects considered. On the other hand, the simplification allows us to decouple the increase in position error due to multiple hops from the influences of geometry.

By taking a very basic repeating pattern as the traffic geometry we can have a setup where all aircraft have the same geometry, but a changing NSE. This allows us to study the largest bridgeable fault for a given LDACS coverage radius and for a given number of aircraft.

For example if aircraft are densely packed in eight tracks, separated from one another by 50 NM along track and a half degree of latitude cross track, as in Fig. 6 (a), it is feasible to bridge a fault of more than $3400 \mathrm{~km}$. This setup can accommodate more than 300 aircraft per track.

The upper extreme shows that the difficulty in coordinating traffic for best A2A positioning performance lies in guaranteeing service with the smallest possible number of participants. To this end we consider a set of three tracks with a spacing of one degree in latitude. We see in Fig. 6 that this approach can cover a distance of $3400 \mathrm{~km}$ with 72 aircraft.

The findings of Fig. 6 support the notion that collaborative positioning works best when the number of participants is highest. In this case number of participants is limited by the separation requirement of the navigation service. The more challenging case is the lower bound: the configuration that will support RNP 4 across the NAT-OTS with the fewest possible number of aircraft. While we are not yet able to produce a guaranteed lower bound, the number 72 does set basic expectations for the order of magnitude of such a bound.

\section{Future Work}

In the process of demonstrating the potential benefits of A2A-enabled positioning for APNT we see two immediate challenges that need addressing: the oceanic interference environment and the intricacies of the co-operative positioning results of Fig. 5 .

The oceanic interference environment is notoriously different from its terrestrial equivalent. A central difference is the lack of terrestrial infrastructure, broadcasting signals in frequency bands reserved for aviation. The reduction 

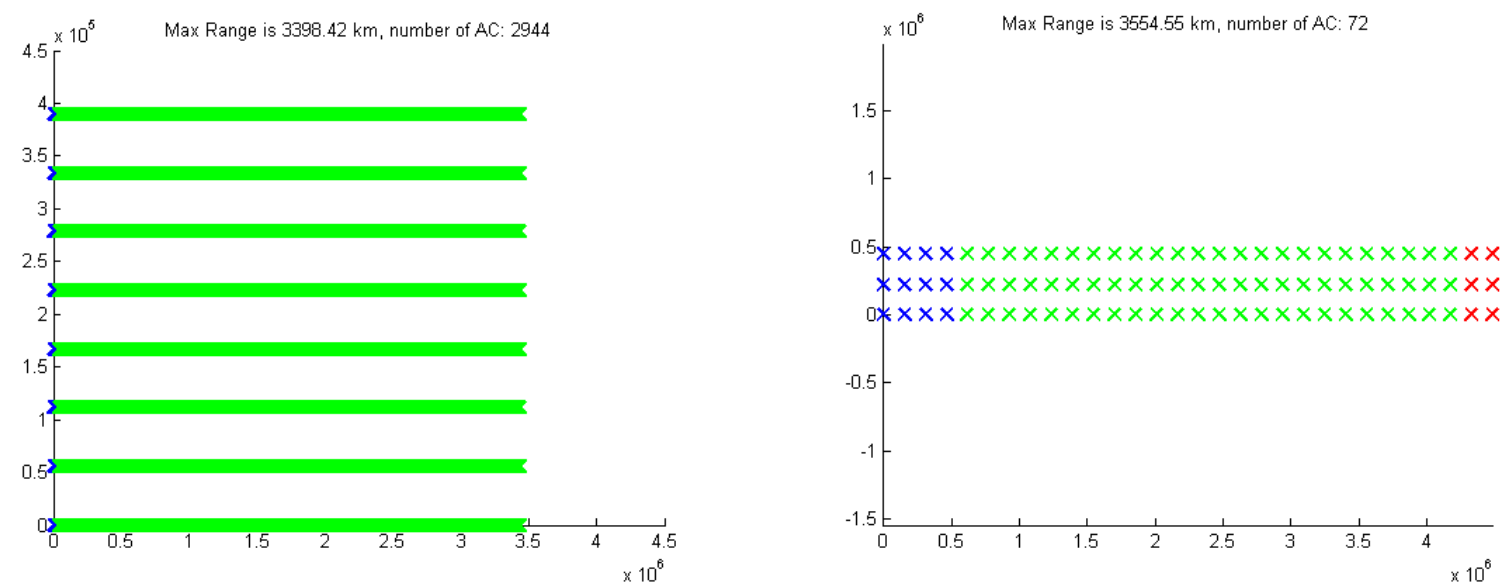

Figure 6: (a) A densely packed NAT-OTS environment would support hundreds of aircraft per track, if A2A ranging became available. (b) A minimum number of aircraft are needed to support service along the entire track, in this case 72 .

in unwanted signal power could result in better ranging performance and increased coverage area. Conversely, the probability of LDACS acting as a disruptor to other systems also decreases, as few other systems are operational during oceanic travel.

The synthetic patterns in which aircraft could potentially fly, as a measure to improve APNT performance, vary by their density. Collaborative positioning works best in the presence of many users, and so the tightest error bounds can be achieved; in the specific case of A2A ranging it appears feasible to support even RNP-1-like operations, if needed. On the other hand, if such density is not needed, it is more desirable to have evenly space aircraft along the entire track system. This trade-space needs to be further investigated, as the effects of coverage distance and aircraft distribution (location and density) on the TSE are complex.

\section{Summary}

This paper picks up the concept of collaborative positioning for civil aviation, using A2A ranging as a way to provide APNT over the North Atlantic Track System. While A2A positioning has been proposed before, its envisioned application had been limited to terminal area operations.

The capability of A2A ranging could be implemented in future versions of LDACS, thus enabling APNT services over oceanic airspace. While the baseline implementation of LDACS-based A2A ranging is not sufficient to cover the entire NAT-OTS, it would likely cover GNSS outages of several thousand kilometers in radius. Providing A2A-based APNT for the NAT-OTS, howver, will require some modifications to LDACS, like increasing the coverag distance. RNP 4 coverage could also be achieved with 4-D trajectories, where required-time-of-arrival services would allow aircraft to fly in specific formations that support higher positioning accuracy.

\section{References}

[1] T. Gräupl. Validating the FACTS2 Air Traffic Simulation Framework. In 2017 Integrated Communications, Navigation and Surveillance Conference (ICNS), pages 1D4-1-1D4-8, April 2017.

[2] EUROCONTROL. Challenges of Growth 2013 - Task 4: European Air Traffic in 2035. Technical report, EUROCONTROL, 2013. 
[3] Okuary Osechas and Gerhard Berz. Improving the Availability of LDACS-Based APNT with Air-to-Air Ranging. In Proc. of the Position, Localization and Navigation Symposium. Savannah, GA, 2016.

[4] ICAO EUR/NAT. North Atlantic Operations and Airspace Manual, seventh edition, 2017.

[5] Thanawat Thiasiriphet, Nicolas Schneckenburger, and Michael Schnell. Ranging With LDACS: Results From Measurement Campaign. In 2016 Integrated Communications Navigation and Surveillance (ICNS), pages 1-18, April 2016.

[6] T Gräupl, M Ehammer, and S Zwettler. L-DACS1 Air-to-Air Data-link Protocol Design and Performance. In Integrated Communications, Navigation and Surveilance Conference (ICNS), 2011, pages B3-1. IEEE, 2011.

[7] Gerhard Berz. ICAO GNSS RFI Mitigation Plan and associated EUROCONTROL Efforts. Technical report, at UN ICG, Nov 2016.

[8] DOC 9613: Performance-Based Navigation (PBN) Manual. Technical report, ICAO, 2008.

[9] Pratap Misra and Per Enge. Global Positioning System: Signals, Measurements and Performance Second Edition. Massachusetts: Ganga-Jamuna Press, 2006.

[10] Thomas Dautermann. Extending required navigation performance to includetime based operations and the vertical dimension. In IEEE ION Position Location and Navigation Symposium, pages 712-720, May 2014.

[11] ICAO EUR/NAT. Future ATM Concept of Operations for the North Atlantic Region, second edition, 2012. 